\title{
SOME NUMERICAL ASPECTS OF THE LEVEL SET METHOD
}

\author{
MATHIAS MOOG, RAINER KECK AND AIVARS ZEMITIS \\ ITWM University of Kaiserslautern \\ Erwin-Schroedinger-Strasse, D-67663 Kaiserslautern, Germany \\ E-mail: moog@itwm.uni-kl.de, rkeck@mathematik.uni-kl.de, \\ zemitis@itwm.uni-kl.de
}

\begin{abstract}
Many practical applications imply the solution of free boundary value problems. If the free boundary is complex and can change its topology, it will be hard to solve such problems numerically. In recent years a new method has been developed, which can handle boundaries with complex geometries. This new method is called the level set method. However, the level set method also has some drawbacks, which are mainly concerning conservation of mass or numerical instabilities of the boundaries.

Our aim is to analyze some aspects of the level set method on the basis of two-phase flow in a Hele-Shaw cell. We investigate instabilities of two-phase flow between two parallel plates. A solution of the linearized problem is obtained analytically in order to check whether the numerical schemes compute reasonable results.

The developed numerical scheme is based on finite difference approximations and the level set method. The equations of two-phase Hele-Shaw flow are written in a modified formulation using the one-dimensional Dirac delta-function. Since the level set function is not smooth enough after re-initialization, special attention during the computation of curvature is needed.

We propose a method that can solve the problems for two-phase Hele-Shaw flow with changing topology. The numerical solution shows good agreement with the analytical solution of the linearized problem. We describe the method below and analyze the results.
\end{abstract}

\section{INTRODUCTION}

Hele-Shaw cells are interesting objects in hydrodynamic and the information about processes there can be important for different applications. Moreover, the simulation of Hele-Shaw cells is a challenging task for numerical methods. Here can be found difficulties which occur also in free boundary value problems for the Navier-Stokes equations. Our experience show that the level set method is harder to use in the case of Hele-Shaw flow as in the case of Stokes flow. Hele-Shaw flow has also similarities with flow in porous media, therefore, new results in this area can give a better understanding of the processes 
in porous materials.

Numerical methods for Hele-Shaw flows are discussed in several papers. An overview is given in [11]. We will concentrate our attention on the instabilities of two-phase flow with different viscosities in Hele-Shaw cells, where the flow is driven by surface tension and a pressure difference. Very often in literature only one of these aspects is analyzed. We are interested in the interaction of surface tension and the viscous forces on the interface, which separates two phases.

If surface tension is taken into account, information about the local curvature $\kappa$ of the interface will be needed. This parameter reinforce the nonlinearity of the free boundary problem for two phase flow. Some analytical estimates for such problems can be achieved by simplifications of the curvature term. The full problem can be solved by numerical methods. Here different approaches can be used. Recent results show, that finite differences in combination with the level set method can compete with methods based on integral equations [9].

We develop a variant of the level set method for Hele-Shaw flow, which can also be used in the case of disruption and coalescence in every phase.

\section{MATHEMATICAL MODEL}

Mathematically, two-phase flow in Hele-Shaw cell can be described by a free boundary value problem. When the speed of motion $\vec{v}$ and the distance between plates $d$ is small enough, then it can be assumed [8], that:

$$
\vec{v}=-K \nabla p
$$

where $p$ is the pressure, $K=\frac{d^{2}}{12 \mu}$ and $\mu$ is the viscosity. In this approximation the process is assumed to be quasi-stationary and at every time step the pressure fulfills an elliptic equation. All equations are assumed to be independent of time. Only the motion of the interface is time-dependent. In the level set approach the interface $\Gamma$ is defined as the zero level of a level set function $F(x, y, t)$ :

$$
\Gamma=\{(x, y): F(x, y, t)=0\} .
$$

In order to investigate the flow in the domain $\bar{\Omega}$, let the level set function divide $\bar{\Omega}$ into two parts $\bar{\Omega}=\overline{\Omega^{+}} \cup \overline{\Omega^{-}}$, where

$$
\begin{aligned}
& \Omega^{+}=\{(x, y) \in \Omega: F(x, y, t)>0\}, \\
& \Omega^{-}=\{(x, y) \in \Omega: F(x, y, t)<0\} .
\end{aligned}
$$

Every part corresponds to an appropriate phase. We denote the outer boundary of the domain $\bar{\Omega}$ by $\partial \Omega$.

Since we consider two phases with different viscosities, the coefficient $K$ has a jump on the interface. Therefore, additional coupling conditions are needed. 
One condition is the continuity of the flux on the interface:

$$
\left[k p_{n}\right]_{\Gamma}=0
$$

and the other is the jump condition for the pressure on the interface, which is given by the Laplace-Young equation:

$$
[p]_{\Gamma}=\sigma \kappa .
$$

Here $p_{n}$ denotes the directional derivative of the pressure in normal direction to the interface, $\sigma$ is the surface tension and $\kappa$ is the curvature of the interface.

We solve the problem in a rectangular domain:

$$
\bar{\Omega}=\{(x, y): x \in[a, b], y \in[c, d]\} .
$$

The standard formulation of the problem can be given as follows:

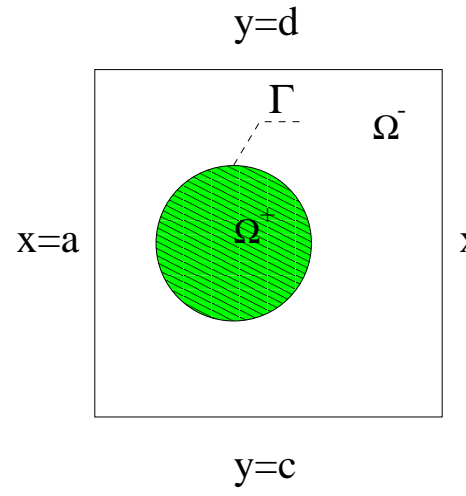

$$
\begin{aligned}
\nabla \cdot(k(x, y) \nabla p) & =0, \text { in } \Omega, \\
{\left[k p_{n}\right]_{\Gamma} } & =0, \\
{[p]_{\Gamma} } & =\sigma \kappa, \\
\left.p\right|_{x=a} & =p_{0}, \\
\left.p\right|_{x=b} & =p_{1}, \\
\left.p_{n}\right|_{y=c} & =0, \\
\left.p_{n}\right|_{y=d} & =0, \\
\partial_{t} F+\vec{v} \cdot \nabla F & =0, \text { in } \Omega, t>0, \\
F(x, y, 0) & =F_{0}(x, y) .
\end{aligned}
$$

Here $p_{0}, p_{1}$ are given constants, $F_{0}(x, y)$ is a given function and

$$
k(x, y)=\left\{\begin{array}{ccc}
k^{+} & \text {if } & (x, y) \in \Omega^{+}, \\
k^{-} & \text {if } & (x, y) \in \Omega^{-} . \\
\Gamma &
\end{array}\right.
$$

The brackets in $[f]_{\Gamma}$ denote the jump on the interface for some function $f$.

\subsection{The modified formulation of the problem}

In [4], a new level set formulation for two-phase flow in the case of NavierStokes equations is given. The idea is to use in the Navier-Stokes equations the one-dimensional Dirac delta function in order to describe the jump condition of the pressure.

Similarly to $[4 ; 10]$ we can get a modified formulation for our problem without explicit jump conditions in the case of porous media

$$
\begin{array}{r}
\nabla \cdot\left(k\left(\nabla p-\alpha \kappa_{F} \delta(F) \nabla F\right)\right)=0, \\
\partial_{t} F+\vec{v} \cdot \nabla F=0 .
\end{array}
$$


where

$$
k(x, y)=k^{-}+\left(k^{+}-k^{-}\right) H(F(x, y, t)) .
$$

Here, $\delta(F)$ is the one-dimensional Dirac delta-function and $H(f)$ is the one-dimensional Heaviside function. The curvature $\kappa_{F}$ in this case is the curvature of the level set function $F(x, y, t)$ at the point $(x, y, t)$. The problem (15)-(16) must be completed with corresponding initial and boundary conditions.

\section{NUMERICAL METHOD}

We will solve the modified problem by finite differences. In the case of explicit time-steeping equation (15) is a linear elliptic equation for the pressure with variable coefficients and it can be approximated by standard difference schemes [7]. If the pressure in known, the speed $\vec{v}$ at every grid-point can also be estimated. These values can be used in equation (16), which is a hyperbolic equation for the level set function $F(x, y, t)$. Therefore, the update in time for the level set function can be done by high-order ENO-schemes [6]. The main steps of the numerical method are the following:

1. Initialization of the level set function

2. Solving of the equation for pressure $p$ and estimation of the velocity $\vec{v}$

3. Update in time of the level set function by the discrete analogous of equation (13)

4. Re-initialization of the level set function

5 . Repeat from step 2.

All steps in the method are very important. The numerical method can work only if these steps are co-ordinated to each other. Since the free boundary is defined by the zero level of the function $F(x, y, t)$, the properties of the discrete level set function are crucial for the method. The function $F$ is only modified during the update-step (step 3) and during re-initialization (step 4). These steps must be implemented so that the following properties can be guaranteed:

- stable motion of the interface

- conservation of mass for every phase

Success of the simulation is guaranteed only if all steps are correctly done. We will explain which stages are most important for the algorithm in our opinion.

\subsection{Calculation of the curvature}

For a discretization of equation (15) the curvature $\kappa_{F}$ must be discretized, too. If we want to use central differences for the approximation of the curvature, the discretized level set function must be smooth enough. Numerical 
experiments with the standard equation for re-initialization (20) show, that even third-order ENO schemes do not guarantee a sufficiently smooth level set function. Therefore, in [4] a special smoothing procedure has been used to enforce smoothness of the level set function. In [5] weighted ENO-schemes (WENO) are investigated and a main result is that these schemes compute smoother solutions then ENO-schemes. We test WENO-schemes and conclude that even then some smoothing is still necessary.

In our approach we do not use a smoothing procedure directly during the update or the re-initialization step. The smoothing is achieved by the calculation of the curvature. We adopt the 'continuous surface force model' from Brackbill, Kothe and Zemach [1] to Hele-Shaw flow.

Near the interface, we compute the curvature with the MAC method, see [1]. The curvature is computed by $\kappa=\nabla \cdot \hat{n}$ with $\hat{n}(x)=\frac{\nabla \phi(x)}{\mid \nabla \phi(x)}$.

In our implementation we store the pressure at the grid points $(i, j)$ and we need the curvature at $\left(i+\frac{1}{2}, j\right)$ and $\left(i, j+\frac{1}{2}\right)$.

$$
\begin{aligned}
n_{i+\frac{1}{2}, j+\frac{1}{2}} & =\left(\begin{array}{c}
\frac{1}{2}\left(\frac{F_{i+1, j}-F_{i, j}}{h_{x}}+\frac{F_{i+1, j+1}-F_{i, j+1}}{h_{x}}\right) \\
\frac{1}{2}\left(\frac{F_{i, j+1}-F_{i, j}}{h_{y}}+\frac{F_{i+1, j+1}-F_{i+1, j}}{h_{y}}\right)
\end{array}\right), \\
n_{i, j} & =\left(\begin{array}{c}
\frac{F_{i+1, j}-F_{i-1, j}}{2 h_{x}} \\
\frac{F_{i, j+1}-F_{i, j-1}}{2 h_{y}} .
\end{array}\right)
\end{aligned}
$$

The normals $n_{i, j}$ can also averaged over the surrounding cell centered normals like in the ALE schemes. The curvature is computed by

$$
\kappa_{i+\frac{1}{2}, j}=\frac{1}{h_{x}}\left(\frac{n_{x i+\frac{1}{2}, j}}{\left|n_{i+\frac{1}{2}, j}\right|}-\frac{n_{x i, j}}{\left|n_{i, j}\right|}\right)+\frac{1}{h_{y}}\left(\frac{n_{x i+\frac{1}{2}, j+\frac{1}{2}}}{\left|n_{i+\frac{1}{2}, j+\frac{1}{2} \mid}\right|}-\frac{n_{x i+\frac{1}{2}, j-\frac{1}{2}}}{\left|n_{i+\frac{1}{2}, j-\frac{1}{2}}\right|}\right) .
$$

\subsection{Re-Initialization (standard way)}

When the update step (16) is performed many times, it may happen, that the gradient of the level set function near the zero level becomes large or small in absolute value. Therefore, re-initialization has to change the gradient of the level set function near the interface without changing its zero level. The standard method for re-initialization is based on solving of the following equation [4] to a steady-state:

$$
\begin{aligned}
\partial_{t} F & =\operatorname{sgn}\left(F_{0}\right)(1-|\nabla F|), \\
F(x, y, 0) & =F_{0}(x, y) .
\end{aligned}
$$

Here, $\operatorname{sgn}(F)$ denotes the sign function. The steady-state solution of this equation has the same zero level as $F_{0}(x, y)$ and it satisfies $|\nabla F|=1$. 
There are many difficulties concerning the numerical solution of the problem (20)-(21). The most important problems are:

- conservation of mass

- smoothness of the function $\mathrm{F}(\mathrm{x}, \mathrm{y}, \mathrm{t})$

Mass will be conserved better if high-order ENO discretization schemes are used for both equations (16) and (20). By our simulations we recognize that even by using high-order ENO schemes mass can be lost. Especially, this may happen if phases change topology. Therefore, we should use some correctionprocedures for mass-conservation. One possibility will be discussed below.

The most important advantage of the level set method is that no explicit information about the interface is needed. The numerical experiments show, that the accuracy of the method cannot be further improved if the position of the zero level is not explicitly used during re-initialization.

\subsection{Extrapolation method for the re-initialization}

We propose a new re-initialization procedure, which leads to better conservation of mass and smoothness the level set function. The main steps are the following:

- the re-initialization is done by equation (20) only on one side of the interface (inside)

- we extrapolate the corresponding values of the level set function which are outside and adjacent to the interface in such a way, that the position of the interface does not change (see figure 1)

This idea can be used in different versions. In one version the position of the interface is found along the coordinate lines. A better method can be achieved, when the position of the zero level is searched and fixed in normal direction to the interface with respect to the surrounding grid points.

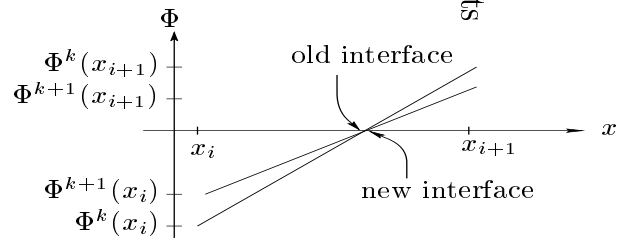

Figure 1. Extrapolated value of the level set function $F^{k+1}\left(x_{i+1}\right)$.

The proposed method gives better mass conservation as the standard reinitialization. In figure 2 one can see, that also the smoothness of the level set function is improved. 

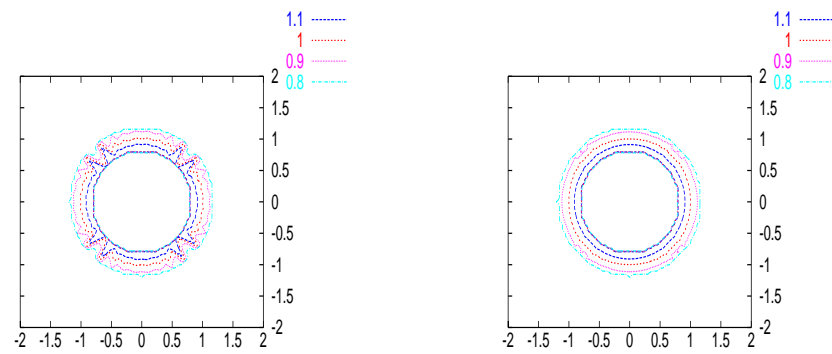

Figure 2. Curvature of the Interface, left: original method, right: modified method

\subsection{Update in time and Mass Conservation}

For the update in time we use equation (16) and an TVD Runge-Kutta method as described in [6]. If the free boundary consists only of one connected contour, mass-conservation can be avoided by using of high-order ENO or WENO schemes. The problems occur after changes in topology. Then a correction of the level set function needed. A very coarse solution is achieved as follows. We add at all points to the level set function a small constant value $\epsilon$. In this case the gradient of the level set function does not change. The constant $\epsilon$ can be chosen in such a way, that the mass after the re-initialization is approximately equal to the mass at the beginning:

$$
\epsilon=\frac{M_{0}-M(t)}{M_{ \pm h}-M(t)} h
$$

Here $M_{0}$ is the mass at the beginning, $M(t)$ is the actual mass, $h$ is the gridconstant, $M_{ \pm h}$ is the mass by adding the value $+h$ to the level set function if $M_{0} \geq M(t)$ and adding $-h$ otherwise.

The mass-correction need not to be done, if the proposed re-initialization procedure is used.

\section{NUMERICAL RESULTS}

The level set method is an universal method. When the update in time and the re-initialization is correctly implemented, then this method can be used for different problems. We have tested the proposed methods with different interface problems in Hele-Shaw Cells and also for 2D Stokes flow. However, in this work we present only solutions for Hele-Shaw flow. 


\subsection{Contraction of a disturbed circle}

The two fluids of viscosity $\mu_{0}$ and $\mu_{1}$ are separated by an interface which is given in cylindrical coordinates by

$$
I(\rho, \varphi, t)=1+\epsilon(t) \sin (n \varphi),
$$

where the inner fluid has the number 1 and the other the number 0 . Our aim is to get an estimation for $\epsilon(t)$. Therefore, we solve a simplified problem, where the curvature is approximated by second order derivatives of the function which defines the interface. The interface conditions are given by the jump in the pressure

$$
[p] \approx \sigma \epsilon(t) n^{2} \sin (n \varphi)
$$

and by the continuity of the velocity in normal direction

$$
\left[v_{\rho}\right]=0 .
$$

We compute the solutions of equations $(6,23,24)$ in an infinite large HeleShaw Cell without external forces

$$
\begin{gathered}
p_{0}=-\frac{\sigma k_{1} n^{2} \epsilon(t) \rho^{-n} \sin (n \varphi)}{k_{0}+k_{1}}, \\
p_{1}=\frac{\sigma k_{0} n^{2} \epsilon(t) \rho^{n} \sin (n \varphi)}{k_{0}+k_{1}} .
\end{gathered}
$$

With an initial value $\epsilon_{0}$ the ordinary differential equation for $\epsilon(t) \frac{\partial I}{\partial t}=\left.v\right|_{\rho=1}$ can be solved, and we obtain a solution:

$$
\begin{aligned}
\epsilon(t) & =\epsilon_{0} e^{-c t}, \\
c & =\frac{\sigma k_{1} k_{0} n^{3}}{k_{0}+k_{1}} .
\end{aligned}
$$

The Interface between two fluids is given by the zero level of the level set function $F$, see figure 3 .

We have compared the estimated contraction rate with our numerical results, see figure 4 . The values of the parameters are $k_{0}=10, k_{1}=1, \sigma=0.001$. In figure 4 one can see the contraction rates for different $n=6,8$ and the theoretical estimates. A rather good agreement can be observed. It is important to remark here, that an unsuitable re-initialization procedure can lead to wrong dependencies for the perturbation $\epsilon$. 

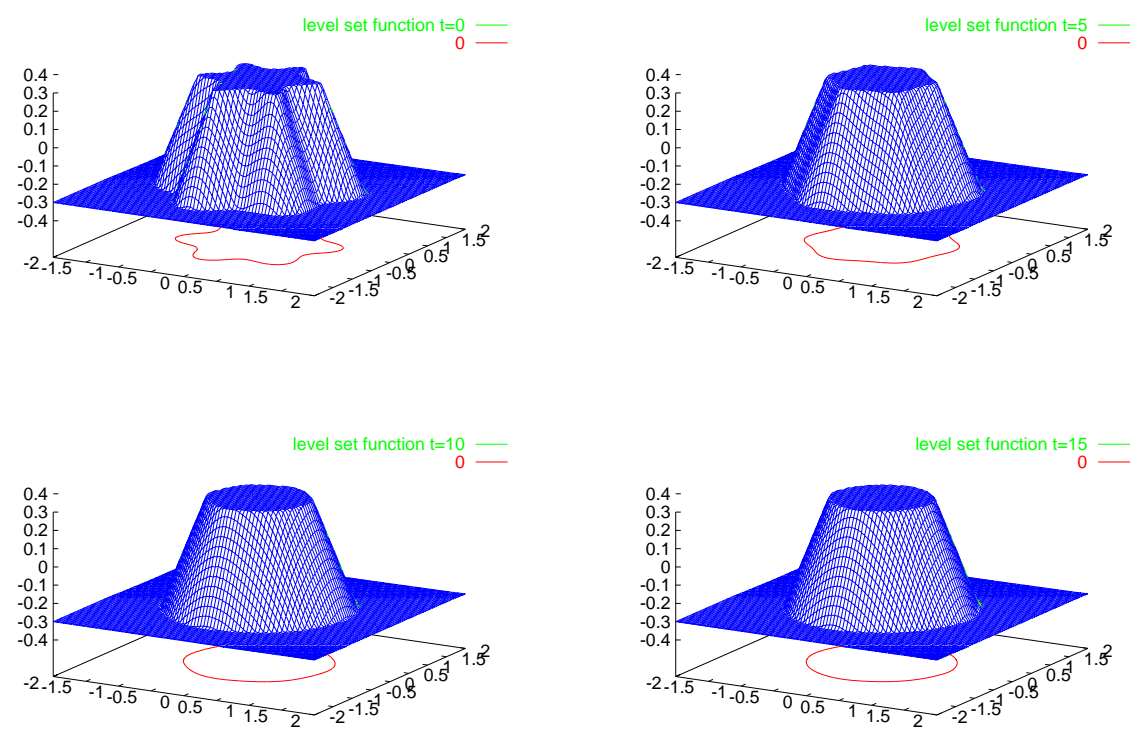

Figure 3. Level Set functions and the zero level for different times $t=0,5,10,25$

\subsection{Instability of small stripes}

Several authors consider the instabilities of small stripes under some initial perturbations, see $[2 ; 3]$. For the theoretical analysis we consider an infinite large Hele-Shaw cell with a given velocity $v_{0}$ in $x$ direction. The two fluids of viscosity $\mu_{0}$ and $\mu_{1}$ are separated by two interfaces

$$
\begin{aligned}
& I^{-}(t)=-d+v_{0} t-a(t) \cos (n y), \\
& I^{+}(t)=d+v_{0} t+b(t) \cos (n y) .
\end{aligned}
$$

where the fluid inside the stripe has the number 1 and the other the number $0 . v_{0}$ is the velocity in $x$ direction at infinity.

The interface conditions are given by the jump in the pressure, where we approximate the curvature by $n^{2} \cos (n y)$,

$$
\begin{aligned}
& {[p]_{I^{-}}=\sigma a(t) n^{2} \cos (n y),} \\
& {[p]_{I^{+}}=\sigma b(t) n^{2} \cos (n y)}
\end{aligned}
$$

and the continuity of the velocity in normal direction

$$
\left[v_{x}\right]_{I^{-}}=0, \quad\left[v_{x}\right]_{I^{+}}=0
$$




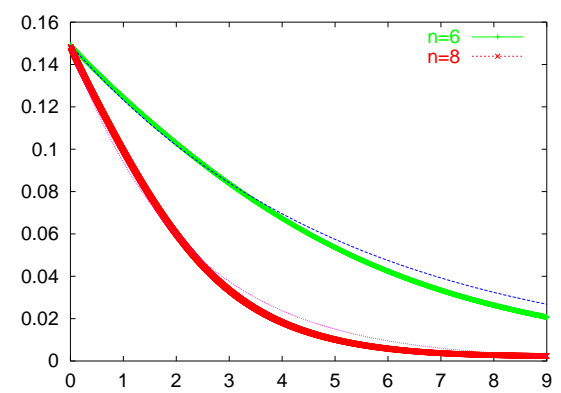

Figure 4. Theoretically (thin lines) and numerically (thick lines) dependence of the disturbance $\epsilon$ in time $\mathrm{t}$ (the grid size is $100 \times 100$ )

We computed the solutions of $(6,31,31)$ with an exponential ansatz using Maple. With an initial value $\alpha$ for $\mathrm{a}(0)$ and $\mathrm{b}(0)$ the system of ordinary differential equations for $a(t)$ and $b(t)$

$$
\begin{aligned}
& v_{0}-\left(\frac{d}{d t} a(t)\right) \cos (k y)=\left.v\right|_{I^{-}}, \\
& v_{0}+\left(\frac{d}{d t} b(t)\right) \cos (k y)=\left.v\right|_{I^{+}}
\end{aligned}
$$

can be solved, and we obtain a solution of the form

$$
\begin{aligned}
& a(t)=\beta e^{-\gamma t}+(\alpha-\beta) e^{\gamma t} \\
& b(t)=\beta e^{\gamma t}+(\alpha-\beta) e^{-\gamma t} .
\end{aligned}
$$

Since the expressions for $\beta$ and $\gamma$ are rather complicate, we plot the solution $\mathrm{a}(\mathrm{t})$ and $\mathrm{b}(\mathrm{t})$ for special parameter values in figure 5 . We observed the same qualitative behavior in our numerical experiments. The amplitude of the disturbance on the left interface increases, while the amplitude of the disturbance on the right interface decreases and changes its sign.

Our analysis is only an approximation for small disturbances. Therefore, we cannot estimate the time of the topology changes.

Using the numerical method we solve the following problem. The domain is defined by $x \in[-2,4]$ and $y \in[-2,2]$. The parameters are as follows: $k_{0}=10$, $k_{1}=1, \sigma=0.0001, n=3, a=b=0.05, d=0.25$. The boundary conditions are given by: $\left.p\right|_{x=-2}=0.2,\left.p\right|_{x=4}=0$, and $\left.\frac{\partial p}{\partial x}\right|_{y= \pm 2}$. The contact angle $\theta$ between the more viscous liquid and the boundaries by $y= \pm 2$ is assumed to be $\theta=95^{\circ}$.

In figure 6 we show the interface at different times. The first figure shows the interface at the beginning. In the second and third figure the disturbance 


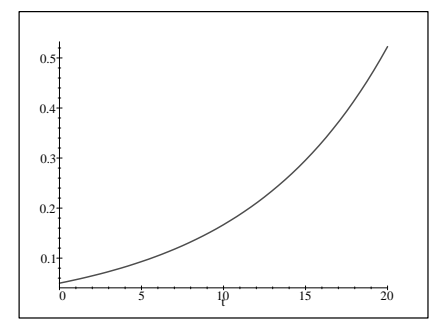

$a(t)$

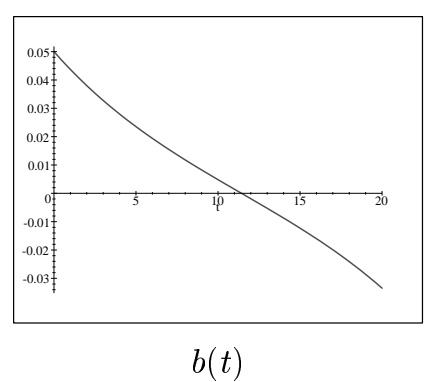

Figure 5. Solutions for $\mathrm{a}$ and $\mathrm{b}$

of the right interface changes its phase relatively to the left interface. The right figure shows the interface after the change in topology.

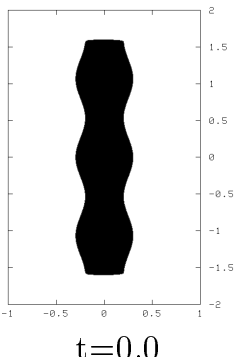

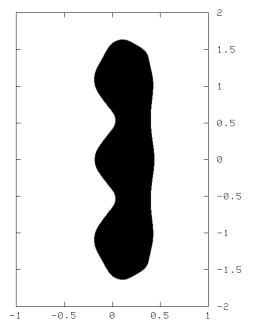

$\mathrm{t}=1.0$

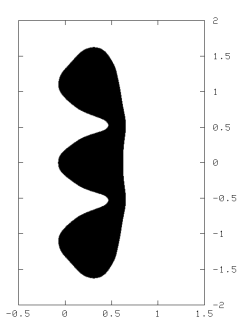

$\mathrm{t}=2.2$

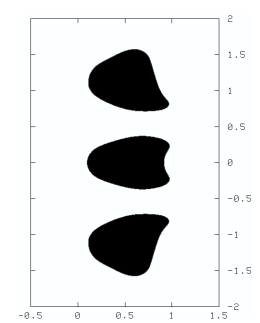

$\mathrm{t}=4.4$

Figure 6. Change of the topology

\subsection{Mass Conservation}

In the experiments with the disturbed circle we observe a good mass conservation, see the left picture in figure 7 . The right picture in that figure shows the behavior of the mass in the experiments with the stripe instabilities. In the case of topological changes we observe a larger loss for the mass but with our mass correction the mass remains constant.

\section{CONCLUSIONS}

In this work a new algorithm based on the level set method for the solution of problems concerning two-phase flow in Hele-Shaw cells has been proposed. One uses a modified formulation of the problem, where the interface between two phases is smoothed.

For Hele-Shaw flow including surface tension effects special attention must be spend to the curvature. Even the usage of high-order ENO or WENO 


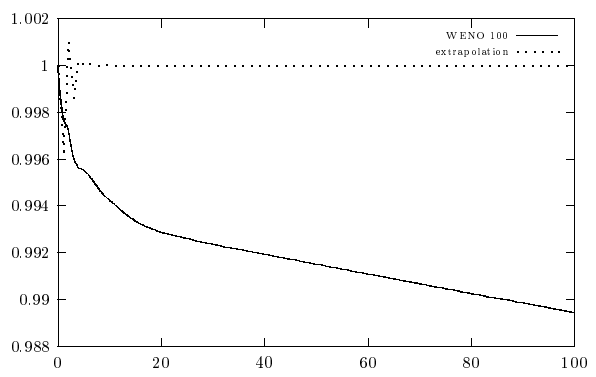

Figure 7. Area loss by conventional re-initialization using equation (20) and the new extrapolation method for the interface. The evolution of area enclosed by the zero level set is plotted over the number of iterations.

schemes does not allow to apply central differences for computing the curvature.

The numerical results are in good agreement with analytical solutions of some linearized problems for Hele-Shaw flows. Formally, the method allows to simulate processes with changing topology, but here additional investigations are also needed.

\section{REFERENCES}

[1] J. U. Brackbill, D.B. Kothe, and C. Zemach. A continuum method for modeling surface tension. Journal of computational Physics, 100:335-354, 1993.

[2] Silvana S.S. Cardoso and Andrew W. Woods. The formation of drops through viscous instability. Journal of Fluid Mechanics, 289, 1995.

[3] A. Cebers. Topological instability of stripes in Hele-Shaw cells 1. Viscous instability. Magnitnaya Gidrodinamika, 33:139-146, 1997.

[4] Y.C. Chang, T.Y. Hou, B. Merriman, and S. Osher. A level set formulation of eulerian interface capturing methods for incompressible fluid flows. Journal of computational Physics, 124:449-464, 1996

[5] Guang-Shan Jiang and Danping Peng. Weighted eno schemes for hamilton-jacobi equations. UCLA CAM Report, 1997.

[6] Stanley Osher and Chi-Wang Shu. High-order essencially nonoscillatory schemes for hamilton-hacobi equations. SIAM Journal on numerical analysis, 28:907-922, 1991.

[7] Aleksandr A. Samarskij. Theorie der Differenzenverfahren. Mathematik und ihre Anwendungen in Physik und Technik; Bd. 40. Leipzig: Geest u. Portig, 1984.

[8] Hermann Schlichting. Boundary-Layer Theory. Series in mechanical engineering. McGraw-Hill, 1979.

[9] Mark Sussman and Peter Smereka. Axisymmetric free boundary problems. Journal of Fluid Mechanics, 341:269-294, 1997.

[10] Mark Sussman, Peter Smereka, and Stanley Osher. A level set aproch for computing solutions to incompressible two-phase flow. Journal of computational Physics, 114:146159, 1994.

[11] Nathaniel Whitaker. Some numerical methods for the Hele-Shaw equations. Journal of Computational Physics, 111:81-88, 1994. 